University of Texas at El Paso

ScholarWorks@UTEP

3-1-2021

\title{
Fuzzy Logic Leads to a More Adequate Way of Processing Likert- Scale Values: Case Study of Burnout
}

\author{
Francisco Zapata \\ The University of Texas at El Paso, fcozpt@outlook.com \\ Olga Kosheleva \\ The University of Texas at El Paso, olgak@utep.edu \\ Vladik Kreinovich \\ The University of Texas at El Paso, vladik@utep.edu
}

Follow this and additional works at: https://scholarworks.utep.edu/cs_techrep

Part of the Computer Sciences Commons

Comments:

Technical Report: UTEP-CS-21-14a

Published in Julia Rayz, Victor Raskin, Scott Dick, and Vladik Kreinovich (eds.), Explainable Al and Other Applications of Fuzzy Techniques,Proceedings of the Annual Conference of the North American Fuzzy Information Processing Society NAFIPS'2021, West Lafayette, Indiana, June 7-9, 2021, Springer, Cham, Switzerland, 2022, pp. 499-504.

\section{Recommended Citation}

Zapata, Francisco; Kosheleva, Olga; and Kreinovich, Vladik, "Fuzzy Logic Leads to a More Adequate Way of Processing Likert-Scale Values: Case Study of Burnout" (2021). Departmental Technical Reports (CS). 1547.

https://scholarworks.utep.edu/cs_techrep/1547

This Article is brought to you for free and open access by the Computer Science at ScholarWorks@UTEP. It has been accepted for inclusion in Departmental Technical Reports (CS) by an authorized administrator of ScholarWorks@UTEP.For more information, please contact Iweber@utep.edu. 


\title{
Fuzzy Logic Leads to a More Adequate Way of Processing Likert-Scale Values: Case Study of Burnout
}

\author{
Francisco Zapata, Olga Kosheleva, and Vladik Kreinovich
}

\begin{abstract}
Many phenomena like burnout are gauged by computing a linear combination of user-provided Likert-scale values. The problem with this traditional approach is that, while it makes sense to have linear combination of weights or other physical characteristics, a linear combination of Likert-scale values like "good" and "satisfactory" does not make sense. The only reason why linear combinations are used in practice is that the corresponding data processing tools are readily available. A more adequate approach would be to use fuzzy $\operatorname{logic}-$ a technique specifically designed to deal with Likert-scale values. We show that fuzzy logic actually leads to a linear combination - but not of the original degrees, but of their transformed values. The corresponding transformation function - as well as the coefficients of the corresponding linear combination - must be determined from the condition that the resulting expression best fits the available data.
\end{abstract}

\section{Formulation of the Problem}

Case study: burnout. In the past, most jobs were menial, tiring jobs. It is possible to get too tired on such a job - in which case the body is no longer capable of continuing, at least not with the same energy. These are natural barriers that evolution came up with - to prevent our bodies from situations when too much physical activity will induce a serious harm to our health.

At present, most jobs have a strong mental, intellectual component. This is a reasonably new phenomenon, for which evolution has not prepared us. In contrast to physical labor, nature does not impose any limits on our mental activities. Even after we are tired, we are still able to do mental work - and many of us continue to do it, especially if there are important deadlines ahead. As a result of this overworking,

Francisco Zapata, Olga Kosheleva, and Vladik Kreinovich

University of Texas at El Paso, 500 W. University, El Paso, TX 79968, USA

e-mail: fcozpt@outlook.com,olgak@utep.edu,vladik@utep.edu 
many people get into what is called burnout - they are stressed, they are unhappy, their productivity plummets. This phenomenon has been observed for many professions; in particular, software companies are affected by this phenomenon.

One of the problems with burnout is that, in contrast to physical tiredness, we ourselves often do not notice it at first, and others do not notice it either - until the situation becomes bad, and a serious intervention is needed.

Since there are no natural indications of a burnout, it is desirable to come up with tools that would allows us to monitor mental workers for such a burnout.

How burnout is measured. Burnout is all about the inner mental state of a person. So, the only reliable way to detect burnout is by asking this person questions - to which a person provides answers of a Likert scale (e.g., from 1 to 5) - and processing the results. This is a typical idea in psychological studies. In particular, for burnout, one of most widely used scheme is the Maslach Burnout Inventory first proposed in [4]. There are also later modifications of this test.

How Likert-scale data is usually processed in psychological analysis: a general description. Usually, a test consists of several $(n)$ questions. For each question $i=1, \ldots, n$, the patient provides an answer $a_{i}$-a number on the corresponding scale. Designers of the test try to find coefficients $c_{1}, \ldots, c_{n}$ for which the corresponding linear combination $c_{1} \cdot a_{1}+\ldots+c_{n} \cdot a_{n}$ is the best correlated with the phenomenon that we are trying to detect.

The resulting linear combination is then used as a numerical measure of the corresponding phenomenon - for example, the measure of burnout.

But why linear combination? Adding kilograms and kilograms makes sense, but adding "good" and "satisfactory" does not. Our understanding is that the only reason for using linear combinations is that this is an available computational tool. Honestly, this is not a very convincing argument.

So what shall we do? What are values on a Likert scale? These are exactly degrees that Lotfi Zadeh started with when he invented fuzzy logic; see, e.g., $[2,3,5,7,8,10]$. So why not use fuzzy logic to process these degrees?

This is what we will explore in this paper.

\section{Analysis of the Problem}

First - naive - idea. Numerous questions on a test describe some symptoms of the analyzed phenomenon - in our case, of a burnout. The more symptoms a patient has, the more severe these symptoms, the more probable it is that this patient is approaching burnout. Each symptom by itself is already some indication of a burnout. So, it seems reasonable to say that a patient has a burnout if this patient has the first symptom or the second symptom, etc. In other words, it seems reasonable to associate the burnout with a disjunction $S_{1} \vee S_{2} \vee \ldots$, where $S_{i}$ means that the $i$-th symptom is present. 
In fuzzy logic, disjunction is represented by an appropriate "or"-operation $f_{\vee}(a, b)$ (also known as a t-conorm). So, it seems reasonable to estimate the degree of a burnout as the value $f_{\vee}\left(a_{1}, a_{2}, \ldots\right)$, where $a_{i}$ is a degree to which the $i$-th symptom is present, and the "or"-operation $f_{\mathrm{V}}(a, b)$ should be determined experimentally, so as to provide the best fit with the available data.

Limitations of the naive idea. The problem with the above idea is that it treats all symptoms equally. In reality, while some symptoms have a strong relation with the analyzed phenomena, the relation of other symptoms with this phenomenon is much weaker.

For example, tiredness is a clear indication of a burnout, while unhappiness may be caused by other reasons - e.g., low salary level, low morale, etc.

So what can we do. So, instead of using the same "or"-operation to combine all the degrees $a_{i}$, we need to take into account that these degrees have different strength: with respect to prediction of the analyzed phenomena, a very strong level of one of the degrees $a_{i}$ may correspond to a much weaker level of another degree $a_{j}$. Thus, before combining, we need to bring these degrees to an equivalent form.

For example, we can bring all these degrees to the level of the degree $a_{1}$. This means that instead of simply combining the original degrees $a_{2}, \ldots$, we first transform them into the $a_{1}$-level, by applying some transformation $f_{i}\left(a_{i}\right)$, and then use the "or"-operation to combine the transformed degree, i.e., to compute the value

$$
a=f_{\vee}\left(a_{1}, f_{2}\left(a_{2}\right), \ldots, f_{i}\left(a_{i}\right), \ldots\right) .
$$

Resulting question: what are these transformations $f_{i}\left(a_{i}\right)$ ? For each symptom $i$, we may (and often do) have several questions describing this symptom. Let us denote the corresponding degrees by $a_{i, 1}, \ldots, a_{i, k}$. In principle, we can deal with this situation in two different ways:

- first idea is to combine these degrees into a single degree $a_{i}=f_{\mathrm{V}}\left(a_{i, 1}, \ldots, a_{i, k}\right)$ and then apply the transformation function $f_{i}\left(a_{i}\right)$ to this combined degree, resulting in the value $f_{i}\left(a_{i}\right)=f_{i}\left(f_{\mathrm{V}}\left(a_{i, 1}, \ldots, a_{i, k}\right)\right)$;

- another idea is to first apply the transformation $f_{i}$ to all $k$ degree, resulting in the values $f_{i}\left(a_{i, 1}\right), \ldots, f_{i}\left(a_{i, k}\right)$, and only then apply the "and"-operation, resulting in the value $f_{\vee}\left(f_{i}\left(a_{i, 1}\right), \ldots, f_{i}\left(a_{i, k}\right)\right)$.

The two resulting values describe the same situation and should, therefore, be equal. This leads to the following definition.

Definition 1. Let $f_{\mathrm{V}}(a, b)$ be an "or"-operation (t-conorm). We say that a function $f(a)$ is consistent with $f_{\vee}$ if for all possible values $a_{1}, \ldots, a_{k}$, we have

$$
f\left(f_{\vee}\left(a_{1}, \ldots, a_{k}\right)\right)=f_{\vee}\left(f\left(a_{1}\right), \ldots, f\left(a_{k}\right)\right) .
$$


Once we have found the appropriate "or"-operation and the appropriate consistent transformation functions $f_{i}\left(a_{i}\right)$, we can then estimate the desired degree $a$ by using the formula (1).

How can we make this mathematical description more practical? It is known that for every "or"-operation $f_{\vee}(a, b)$, and for every $\varepsilon>0$, there exists an $\varepsilon$-close strictly Archimedean "or"-operation, i.e., operation of the form

$$
F^{-1}(F(a)+F(b))
$$

for some monotonic continuous function $F(a)$, where $F^{-1}(v)$ denotes the inverse function: $a=F^{-1}(v) \Leftrightarrow v=F(a)$; see, e.g., [6]. For very small $\varepsilon$, the difference between these two operations can be safely ignored. So, for all practical purposes, it makes sense to assume that the actual "or"-operation has the form (3).

Under this assumption, we can get a full description of all possible consistent transformations.

Proposition 1. For each "or"-operation of type (3), every function $f(a)$ which is consistent with this operation has the form

$$
f(a)=F^{-1}(c \cdot F(a))
$$

for some constant $c>0$.

Proof. Substituting the expression (3) into the formula (2), we conclude that

$$
f\left(F^{-1}\left(F\left(a_{1}\right)+\ldots+F\left(a_{k}\right)\right)\right)=F^{-1}\left(F\left(f\left(a_{1}\right)\right)+\ldots+F\left(f\left(a_{k}\right)\right)\right) .
$$

By applying the function $F(a)$ to both sides of this equality, we conclude that

$$
F\left(f\left(F^{-1}\left(F\left(a_{1}\right)+\ldots+F\left(a_{k}\right)\right)\right)\right)=F\left(f\left(a_{1}\right)\right)+\ldots+F\left(f\left(a_{k}\right)\right) .
$$

Let us denote $G(a) \stackrel{\text { def }}{=} F\left(f\left(F^{-1}(a)\right)\right)$, then the left-hand side of the formula (6) takes the form $G\left(F\left(a_{1}\right)+\ldots+F\left(a_{k}\right)\right)$, while each term in the right-hand side takes the form

$$
F\left(f\left(a_{i}\right)\right)=F\left(f\left(F^{-1}\left(F\left(a_{i}\right)\right)\right)\right)=G\left(F\left(a_{i}\right)\right) .
$$

Thus, the formula (6) takes the form

$$
G\left(F\left(a_{1}\right)+\ldots+F\left(a_{k}\right)\right)=G\left(F\left(a_{1}\right)\right)+\ldots+G\left(F\left(a_{k}\right)\right) .
$$

For each set of values $v_{i}$, we can take $a_{i}=F^{-1}\left(v_{i}\right)$, then $F\left(a_{i}\right)=v_{i}$, so the formula (8) takes the form

$$
G\left(v_{1}+\ldots+v_{k}\right)=G\left(v_{1}\right)+\ldots+G\left(v_{k}\right) .
$$

For a monotonic function $G(v)$, this implies that the function $G(v)$ has the form $F(v)=c \cdot v$ for some constant $c$; see, e.g., [1]. By definition of the function $G(v)$, this means that $F\left(f\left(F^{-1}(v)\right)\right)=c \cdot v$. So, for $a=f^{-1}(v)$, for which $v=F(a)$, we 
get

$$
F(f(a))=c \cdot F(a) .
$$

By applying the inverse function $F^{-1}$ to both sides of the formula (9), we get the desired expression (4).

The proposition is proven.

Resulting combination rule. If we substitute the expression (3) for the "or"operation into the formula (1), we get

$$
a=F^{-1}\left(F\left(a_{1}\right)+F\left(f_{2}\left(a_{2}\right)\right)+\ldots+F\left(f_{i}\left(a_{i}\right)\right)+\ldots\right),
$$

i.e., equivalently,

$$
F(a)=F\left(a_{1}\right)+F\left(f_{2}\left(a_{2}\right)\right)+\ldots+F\left(f_{i}\left(a_{i}\right)\right)+\ldots
$$

To make this formula uniform, we can define $f_{1}\left(a_{1}\right)=a_{1}$, then this formula takes the form

$$
F(a)=F\left(f_{1}\left(a_{1}\right)\right)+F\left(f_{2}\left(a_{2}\right)\right)+\ldots+F\left(f_{i}\left(a_{i}\right)\right)+\ldots
$$

According to the formula (9) from the above Proposition, for each $i$, we have

$$
F\left(f_{i}\left(a_{i}\right)\right)=c_{i} \cdot F\left(a_{i}\right)
$$

for some constant $c_{i}$. Substituting the expression (11) into the formula (10), we conclude that

$$
F(a)=c_{1} \cdot F\left(a_{1}\right)+c_{2} \cdot F\left(a_{2}\right)+\ldots+c_{i} \cdot F\left(a_{i}\right)+\ldots
$$

Discussion. Our goal is to find the severity of the burnout - or of any other tested phenomenon.

From this viewpoint, what is important are not so much the numerical values themselves, but the relation between values corresponding to different situation: which is larger and which is smaller. Since the function $F(a)$ is monotonic, we can therefore use $A \stackrel{\text { def }}{=} F(a)$ instead of $a$.

So, we arrive at the following recommendation.

\section{Resulting Recommendation}

Recommendation. Once we have the degrees $a_{1}, \ldots, a_{n}$ corresponding to different questions, the overall degree $A$ can be estimated as

$$
A=c_{1} \cdot F\left(a_{1}\right)+\ldots+c_{n} \cdot F\left(a_{n}\right),
$$


where the values $c_{i}$ and the function $F(a)$ should be determined based on the available data: they should provide the best fit for the cases when the value $A$ is known.

Comments.

- We still get a linear combination - but a linear combination after some possibly non-linear transformation $F(a)$. The main advantage is that now we have a justification, so a linear combination is not just a heuristic idea.

- Our preliminary results show that by appropriately selecting a function $F(a)$, we can indeed get a better fit that simply by using a linear combination of values $a_{i}$.

- Similar results can be obtained if we allow patients to provide interval ranges $\left[\underline{a}_{i}, \bar{a}_{i}\right]$. In this case, instead of a single value $A$, we get the while range $[\underline{A}, \bar{A}]$ of values corresponding to different values $a_{i}$ from the corresponding intervals. Due to monotonicity, the smallest value of $A$ is attained when all the values $a_{i}$ are the smallest possible, and the largest value of $A$ is attained when all the values $a_{i}$ are the largest possible:

$$
\begin{aligned}
& \underline{A}=c_{1} \cdot F\left(\underline{a}_{1}\right)+\ldots+c_{n} \cdot F\left(\underline{a}_{n}\right) ; \\
& \bar{A}=c_{1} \cdot F\left(\bar{a}_{1}\right)+\ldots+c_{n} \cdot F\left(\bar{a}_{n}\right) .
\end{aligned}
$$

\section{Acknowledgments}

This work was supported in part by the National Science Foundation grants 1623190 (A Model of Change for Preparing a New Generation for Professional Practice in Computer Science), and HRD-1834620 and HRD-2034030 (CAHSI Includes). It was also supported by the program of the development of the Scientific-Educational Mathematical Center of Volga Federal District No. 075-02-2020-1478.

The authors are thankful to the anonymous referees for valuable suggestions.

\section{References}

1. J. Aczél and J. Dhombres, Functional Equations in Several Variables, Cambridge University Press, 2008.

2. R. Belohlavek, J. W. Dauben, and G. J. Klir, Fuzzy Logic and Mathematics: A Historical Perspective, Oxford University Press, New York, 2017.

3. G. Klir and B. Yuan, Fuzzy Sets and Fuzzy Logic, Prentice Hall, Upper Saddle River, New Jersey, 1995.

4. C. Maslach and S. E. Jackson, "The measurement of experienced burnout", Journal of Occupational Behavior, 1981, Vol. 2, No. 2, pp. 99-113.

5. J. M. Mendel, Uncertain Rule-Based Fuzzy Systems: Introduction and New Directions, Springer, Cham, Switzerland, 2017. 
6. H. T. Nguyen, V. Kreinovich, and P. Wojciechowski, "Strict Archimedean t-norms and tvonorms as universal approximators", International Journal of Approximate Reasoning, 1998, Vol. 18, Nos. 3-4, pp. 239-249.

7. H. T. Nguyen, C. L. Walker, and E. A. Walker, A First Course in Fuzzy Logic, Chapman and Hall/CRC, Boca Raton, Florida, 2019.

8. V. Novák, I. Perfilieva, and J. Močkoř, Mathematical Principles of Fuzzy Logic, Kluwer, Boston, Dordrecht, 1999.

9. S. G. Rabinovich, Measurement Errors and Uncertainties: Theory and Practice, Springer, New York, 2005.

10. L. A. Zadeh, "Fuzzy sets", Information and Control, 1965, Vol. 8, pp. 338-353. 